\title{
The importance of molecular diagnostics for infectious diseases in low-resource settings
}

Iruka N. Okeke $\mathbb{B}^{1 凶}$ and Chikwe Ihekweazu ${ }^{2}$

In settings with limited resources and a wide range of possible etiologies, molecular technologies offer an effective solution for infectious disease diagnostics, because they are agile, fast and flexible. Health systems that routinely use molecular diagnostics will achieve economies of scale, maximize limited expertize and rapidly respond to new threats.

A golden rule for building laboratory capacity in resource-limited settings is selecting techniques that are workable and can be sustained. Despite their utility and precision, molecular diagnostics were deemed 'inappropriate' for decades, as they were considered to be too finicky, contamination-prone, expensive and technically complex to be used where resources are constrained, skills in short supply and infrastructure not up to date. However, traditional culture-based clinical microbiology, typically deemed 'appropriate', has been notoriously difficult to sustain at the required quality in lowest-income settings without external support ${ }^{1,2}$. Some pathogens are cheap to culture, whereas culture is costly for others. The impact of culture-based methods on patient care, infection prevention and control, and outbreak responses is further limited by long turnaround times and challenges in quality assurance. Serological methods, although typically much faster and simpler, often do not provide the breadth and depth of information needed to make informed and effective decisions, sometimes lack specificity and are not available for many pathogens. Supply chains for pathogen-specific diagnostics are also complex, easily disrupted and difficult to adjust to new pathogens.

Most African countries not only face limited resources but also a high burden and variety of infectious diseases. Fever, the most common symptom leading to attendance of a health-care institution in Nigeria, can arise from infection by $>200$ different pathogens, including malaria parasites, Salmonella enterica and a range of hemorrhagic viruses. Many of the life-threatening pathogens cannot be reliably detected by culture without expensive additional instrumentation, tissue culture or biosafety upgrades. This combination of broad pathogen range and resource constraints, also shared by many high-infectious disease burden settings in South Asia and South America, highlights the need for efficient molecular diagnostics.

The preference for 'simple' culture-based methods comes from a focus on single diseases and is often motivated and paid for through research but unsuitable to serve broad health system needs. By offering more diagnostic precision for a broad pathogen range, using a small consumable palette, molecular diagnostics are cost-effective, in spite of their high upfront expense, and flexible enough to pivot rapidly to new threats ${ }^{3}$. In resource-limited settings molecular equipment is commonly reserved for special or reference-only testing. But broader application of these resources will produce economies of scale and make local production of testing materials commercially viable in the low-income countries that need them most.

But how feasible is it to introduce molecular diagnostics into a setting with a limited budget, poor infrastructure and few skilled personnel? COVID-19 provides a practical proof-of concept. Between January 2020 and January 2021, the number of public health laboratories in Nigeria equipped to undertake molecular identification of SARS-CoV-2 in clinical specimens increased from four to 72 . This increase was accomplished by exemplary coordination and oversight, tiered training and pooled procurement. Ancillary services, such as biosafety, supply chain management, bioengineering and quality assurance, were expanded in parallel. Similar exponential growth in public health testing capacity

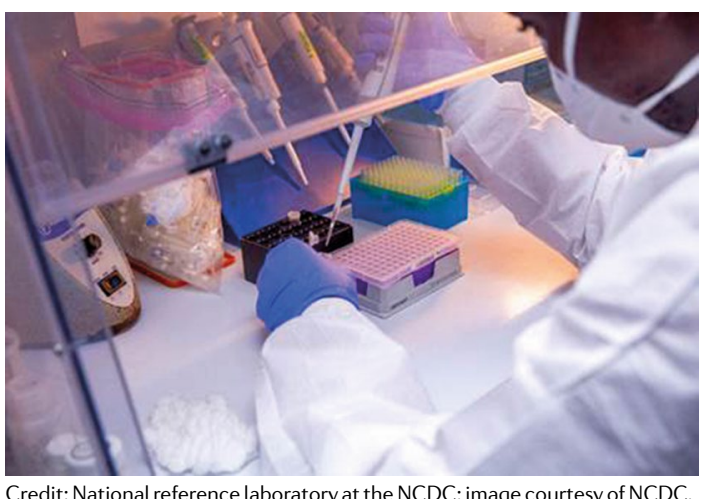


has been reported from other LMIC settings. Moving quickly to solidify and build on these gains is the best way to pay forward substantial COVID-19 diagnostic investments to other infectious diseases ${ }^{4}$.

Success case studies for molecular diagnostic applications in low-income settings extend well beyond COVID-19. Unexpected appearance of Ebola and Yellow fever hemorrhagic viruses in Nigeria in the past decade was rapidly detected using molecular methods weeks before more standard reference techniques could be deployed. In both instances, the advance information provided by on-the-spot PCR testing provided early insights that guided the public health response ${ }^{3}$. Culture followed by whole-genome sequencing (WGS) made it possible to simultaneously identify the unusual etiologic agents of neonatal sepsis in hospitals in the Gambia, determine their antimicrobial resistance profiles and pinpoint nosocomial outbreaks ${ }^{5}$. Molecular methods can increase pathogen identification in patients with meningitis several-fold, as shown by the examination of archived samples from Bangladesh ${ }^{6}$, which trebled the conventional sensitivity and retrospectively uncovered a chikungunya virus outbreak. These and many other examples build a strong case for routine use of molecular diagnostics even in the face of, largely surmountable, challenges ${ }^{7}$.

As has been stated before, "PCR is an easy thing to do badly" , and the likelihood of waste, contamination or data misinterpretations from poorly executed molecular tests are the nightmares of even the most forward-thinking diagnostic advocates. WGS might mitigate some PCR limitations. There is a pressing need to expand and solidify the molecular biology knowledge-base in low-income countries and to scale up access to external quality assurance. Microfluidics could protect sensitive steps from error, and miniaturization will save on expensive reagents ${ }^{2}$. Deployment of Gene Xpert for the detection of tuberculosis in resource-constrained settings is a case in point ${ }^{8}$ and was leveraged for Ebola and SARS-CoV-2 outbreak testing.

Molecular diagnostics will not, and should not, displace culture any time soon but will provide access to testing for millions that do not have it now. There is huge potential to revolutionize surveillance of hard-toreach populations and to cover large ground with few resources. For example, detecting feco-orally transmitted pathogens can help to identify transmission hotspots and to target vaccine campaigns. In the case of typhoid, the routine diagnostic, blood culture, has poor sensitivity and culture from environmental sources is even less sensitive. But detecting S. enterica and other pathogens by DNA-based methods is simple and effective and has successfully been used to map typhoid hot spots ${ }^{9}$ as well as to close in on polioviruses in the eradication end-game ${ }^{10}$. Analogous methods can be used for cholera, enteric viruses, and even SARS-CoV-2.
The present promises of molecular diagnostics are a tip of the ice berg, and now is the time to address the hitches that prevent expansion of their use. Pandemicinspired emergency solutions to supply chain failures that plague low-income country health systems and laboratories need to be replaced with robust procurement, shipping and handling, stock management and waste disposal systems. Many laboratories have now installed solar back-ups to overcome frequent power supply interruptions. 'Smart' machines that can seamlessly resume after a power interruption are those that will feature in the low-resource but fastest-growing diagnostic landscapes of the next decade. Next generation, truly appropriate equipment, already on the market for some applications (for example Oxford nanopore sequencers), will require only intermittent power supply. Creative solutions to other infrastructure and supply chain gaps must come from outside biomedicine as well. National public health institutes are best positioned to provide much-needed governance, coordination and support for the expansion of public health diagnostics and have already shown leadership in multiple contexts.

We can and should innovate to fit molecular diagnostics for purpose, rather than work around the reluctance to tailor them for infrastructure-poor settings that need them most.

1. Ombelet, S. et al. Clinical bacteriology in low-resource settings: today's solutions. Lancet Infect. Dis. 18, e248-e258 (2018).

2. Okeke, I. N. et al. Leapfrogging laboratories: the promise and pitfalls of high-tech solutions for antimicrobial resistance surveillance in low-income settings. BMJ Glob. Health 5, e003622 (2020).

3. Naidoo, D. \& Ihekweazu, C. Nigeria's efforts to strengthen laboratory diagnostics - why access to reliable and affordable diagnostics is key to building resilient laboratory systems. Afr. J. Lab. Med. 9, 1019 (2020).

4. Saha, S. \& Pai, M. Can COVID-19 innovations and systems help low- and middle-income countries to re-imagine healthcare delivery? Med (N.Y.) 2, 369-373 (2021).

5. Okomo, U. et al. Investigation of sequential outbreaks of Burkholderia cepacia and multidrug-resistant extended spectrum $\beta$-lactamase producing Klebsiella species in a West African tertiary hospital neonatal unit: a retrospective genomic analysis. Lancet Microbe 1, e119-e129 (2020).

6. Saha, S. et al. Unbiased metagenomic sequencing for pediatric meningitis in Bangladesh reveals neuroinvasive chikungunya virus outbreak and other unrealized pathogens. mBio 10, e02877-19 (2019).

7. Kelly-Cirino, C. D. et al. Importance of diagnostics in epidemic and pandemic preparedness. BMJ Glob. Health 4, e001179 (2019).

8. Albert, H. et al. Development, roll-out and impact of Xpert MTB/RIF for tuberculosis: what lessons have we learnt and how can we do better? Eur. Respir. J. 48, 516-525 (2016).

9. Andrews, J. R. et al. Environmental surveillance as a tool for identifying high-risk settings for typhoid transmission. Clin. Infect. Dis. 71, S71-S78 (2020).

10. Johnson Muluh, T. et al. Contribution of environmental surveillance toward interruption of poliovirus transmission in Nigeria 2012-2015. J. Infect. Dis. 213, S131-S135 (2016).

Competing interests

The authors declare no competing interests. 